Rev. Int. Contam. Ambie. 33 (Especial Biotecnología e ingeniería ambiental) 43-51, 2017

DOI: 10.20937/RICA.2017.33.esp01.04

\title{
INSTRUMENTOS TECNOLÓGICOS PATENTADOS EN MÉXICO PARA TRATAR AGUAS RESIDUALES
}

\author{
Lilian Edith DOMÍNGUEZ-MONTERO ${ }^{1}$, Héctor Mario POGGI-VARALDO ${ }^{1,2 *}$, \\ Miguel Ángel PÉREZ-ANGÓN ${ }^{1}$, Blanca Elena JIMÉNEZ-CISNEROS ${ }^{3}$, \\ Rosa Olivia CAÑIZARES-VILLANUEVA ${ }^{1}$, Sergio CAFFAREL-MÉNDEZ ${ }^{4}$ y \\ Eugenio FRIXIONE-GARDUÑO ${ }^{1}$
}

${ }^{1}$ Programa Transdisciplinario en Desarrollo Científico y Tecnológico para la Sociedad, Centro de Investigación y de Estudios Avanzados, Instituto Politécnico Nacional. Avenida Instituto Politécnico Nacional 2508, Gustavo A. Madero, San Pedro Zacatenco, Ciudad de México, México, C.P. 07360

${ }^{2}$ Departamento de Biotecnología y Bioingeniería, Centro de Investigación y de Estudios Avanzados, Instituto Politécnico Nacional. Avenida Instituto Politécnico Nacional 2508, Gustavo A. Madero, San Pedro Zacatenco, Ciudad de México, México, C.P. 07360

${ }^{3}$ Departamento de Ingeniería Ambiental, Instituto de Ingeniería, Universidad Nacional Autónoma de México. Circuito escolar s/n, Ciudad Universitaria, Delegación Coyoacán, Ciudad de México, México, C.P. 04510

${ }^{4}$ División de Ingeniería Química y Bioquímica, Tecnológico de Estudios Superiores de Ecatepec. Avenida Campo de Mayo s/n, Ecatepec, Estado de México, México, C.P. 56100

*Autor para correspondencia: r4cepe@yahoo.com

(Recibido marzo 2015; aceptado octubre 2015)

Palabras clave: México, patentes, tecnología

\section{RESUMEN}

El desarrollo y adaptación de tecnología es de vital importancia para que el tratamiento de aguas residuales (TAR) sea eficiente, fácil de implementar y con bajos costos de construcción y operación. Las patentes pueden ser un indicador útil para medir y analizar dichos avances tecnológicos. El propósito de este trabajo fue evaluar cómo ha evolucionado el registro de la concesión de patentes en el sector TAR. En particular, se analizó la tecnología patentada en México por titulares extranjeros y mexicanos. La revisión de bases de datos de patentes relacionados con el TAR consideró los últimos 18 años teniendo en cuenta que las principales normas de aguas residuales datan desde 1996. Las bases de datos examinadas fueron las del Instituto Mexicano de la Propiedad Industrial (IMPI) y de la Organización Mundial de la Propiedad Intelectual (OMPI). Los resultados muestran que el volumen de la tecnología patentada en México para el TAR por titulares extranjeros y mexicanos es escaso en comparación con el volumen de patentes correspondientes a otros países del mundo. México sólo ha contribuido con 34 patentes de un total de 5041 (0.67 \%) en el periodo de 1996 a 2013, según la OMPI, de la cuales siete pertenecen a residentes mexicanos. Además, se determinó que la tecnología en patentes mexicanas es una proporción menor de todas las patentes en TAR. De 1996 a 2013, en la base de datos del IMPI se registraron 137 patentes, de las cuales 42 pertenecen a empresas mexicanas, universidades, centros de investigación e inventores particulares (30.65\%). Esto sugiere que el núcleo endógeno de invención de tecnología para el sector TAR en México es marginal y no hay evidencia de que esté vinculado con el flujo global en la materia. 
Key words: Mexico, patents, technology

\begin{abstract}
The development and adaptation of technology is vital for wastewater treatment (WWT) to be efficient, easy to implement, and with low costs of construction and operation. Patents can be a useful indicator for measuring and analyzing these technological issues. The purpose of this study was to evaluate how the registration on granted of patents in the WWT sector has evolved. In particular, patented technology in Mexico by foreign and Mexican holders was analyzed. The Mexican Institute of Industrial Property (IMPI, in spanish) and the World Intellectual Property Organization (WIPO) databases were examined for a period of 18 years, taking into account that the enforcement of wastewater standards began since 1996. The results show that the volume of patented technology in Mexico for WWT by foreign and Mexican holders is scarce compared to the volume of patents for other countries in the world. Mexico has contributed with only 34 patents out of $5041(0.67 \%)$ in the period 1996 to 2013 according to the WIPO dababase; seven patents belong to Mexican residents. Furthermore, it was determined that the technology in Mexican patents is a minor proportion of all the patents in WWT. Since 1996 to 2013, in the database of IMPI, there were registered 137 patents, of which 42 belong to Mexican companies, institutions, research centers and inventors $(30.65 \%)$. This suggests that the endogenous nucleus of technology invention for the WWT sector in Mexico is marginal and there is no evidence of linkage with the global flow in the matter.
\end{abstract}

\section{INTRODUCCIÓN}

El agua es uno de los recursos naturales indispensable para el desarrollo de la vida, además de ser parte importante en el desarrollo de cualquier país, su disponibilidad ha decrecido debido a la contaminación que se ha suscitado por diversos medios (Romero-Aguilar et al. 2009).

En México, la contaminación del agua se debe a la inadecuada disposición de las aguas residuales de origen municipal, industrial y de naturaleza variada, como el drenaje agrícola, la escorrentía pluvial, entre otras (Jiménez et al. 2010). Las aguas residuales municipales que se generan en México están compuestas principalmente de nutrientes (nitrógeno y fósforo), cantidades traza de compuestos orgánicos recalcitrantes y metales (Salgado-Bernal et al. 2012). En 2011, México generó $210.1 \mathrm{~m}^{3} / \mathrm{s}$ de aguas residuales municipales y se trataron solamente $46.5 \%$ de ellas (Domínguez-Montero, com. pers.), lo que sigue propiciando el reúso de agua residual bruta para riego (Jiménez 2006).

El tratamiento de aguas residuales (TAR) puede ser un proceso productivo del cual se obtiene agua tratada, siendo una de sus finalidades la mejora del ambiente y la reducción de aguas contaminadas (De la Peña et al. 2013). Por lo que hoy en día se cuenta con diversas tecnologías altamente mecanizadas e incluso tecnologías "apropiadas" de bajo costo para lograr el TAR (Zurita-Martínez et al. 2011).

Para conocer los cambios que han resultado de la innovación tecnológica, es necesario realizar una vigilancia tecnológica, la cual es un proceso para captar la información sobre tecnología, seleccionarla, analizarla y difundirla para convertirla en conocimiento (Delgado et al. 2010)

Un estudio estratégico para la vigilancia tecnológica en un campo dado se realiza a través de la información de patentes, ya que permite conocer la actividad inventiva de la sociedad mundial (Vidal 2008). Una patente, de acuerdo con la Organización Mundial de la Propiedad Intelectual es "un derecho exclusivo concedido a una invención, es decir, un producto o procedimiento que aporta, en general, una nueva manera de hacer algo o una nueva solución técnica a un problema" (OMPI 2014a). Además, una patente cuenta con registros completos con información tecnológica y de más largo alcance en términos históricos y es una de las propiedades intelectuales más importantes hasta el momento (Aboites y Soria 1999).

México cuenta con la Ley de Propiedad Industrial (LPI) y el Instituto Mexicano de Propiedad Intelectual (IMPI) que se encarga de ejecutar y cumplir la LPI. Además, el IMPI se encarga de la protección de los derechos de propiedad industrial únicamente en 
el país donde ésta es solicitada y concedida (IMPI 2014b).

México es uno de los 184 países asociados a la Organización Mundial de la Protección Industrial (OMPI) y al Tratado de Cooperación en Materia de Patentes (PCT, por sus siglas en inglés). El principal objetivo del PCT es el de simplificar (hacer más eficaz y económico) el procedimiento a seguir para solicitar la protección de una patente de invención en varios países. La OMPI y el PCT no son los encargados de otorgar la concesión de patentes internacionales, dicha tarea y responsabilidad le compete de manera exclusiva a cada una de las oficinas de patentes de los países donde se solicita la protección (IMPI 2014c). Por ello, los inventores nacionales desde un principio pueden registrar una solicitud nacional e internacional, con el propósito de tener mayor competencia mundial (Vidal 2008).

Aboites y Soria (1999) por medio de las bases de datos que contienen los registros de las patentes han realizado el análisis de algunos sectores tecnológicos en México. Para conocer el desarrollo de la innovación, han comparado la situación de patentamiento con otros países, principalmente Estados Unidos. Además han analizado el eje de patentamiento de residentes y no residentes. Ramos Muñiz et al. (2005) y García y Corvo (2007) también han estudiado la relación del desarrollo tecnológico y su comportamiento por medio de la información contenida en documentos de patentes.

Para el caso de TAR en México, Ramírez Guardado et al. (2013) realizaron el análisis de patentes para equipos estratégicos de la Planta Cerro de la Estrella debido a que es un indicador que permite tener una vigilancia de los avances tecnológicos respecto a las patentes anteriores, por lo que se crea una trayectoria tecnológica.

Por ello, hemos expuesto la hipótesis de que México cuenta con un número limitado de patentes desarrolladas para el TAR y en su mayoría no pertenecen a titulares mexicanos. Sin embargo, hasta ahora no existe un estudio sobre el estatus tecnológico del TAR en México que haya utilizado el método de estudio de patentes u otros métodos. En este sentido, el objetivo de este trabajo es presentar cómo ha evolucionado el sector TAR en la concesión de patentes en México y en el mundo entre 1996 y 2013.

\section{MATERIALES Y MÉTODOS}

El método utilizado se centró en la estrategia de búsqueda trazada, que tuvo como premisa la definición del objeto, objetivo y la estrategia de búsqueda, a saber:
Objetivo de la búsqueda: evaluar cómo ha evolucionado en el sector TAR, entre 1996 y 2013, en el registro de patentes concedidas a titulares extranjeros y mexicanos tanto en México (IMPI) como desde México a través del PCT (OMPI).

Objeto de búsqueda: TAR.

Las fuentes de información que conformaron este estudio fueron dos bases de datos de documentos de patentes de libre acceso en internet, la primera fue la base de la OMPI y la segunda la del IMPI.

En la base de datos patentscope del OMPI a través de la sección de búsqueda (research) se realizó la exploración de información por medio de la combinación de campo (field combination) en donde se pidió el término "wastewater (agua residual)" en la sección de "título en inglés (english title)" y en "resumen en inglés (english abstract)". En la sección de "fecha de publicación (publication date)" se colocaron las fechas de 01-01-1996 al 31-12-2013.

En la base de datos del Sistema de Información de la Gaceta de la Propiedad Industrial (SIGA-IMPI) a través de una búsqueda simple se eligió la opción de "patente". El término que se buscó fue "aguas residuales". El sistema de dicha base de datos arroja los resultados en fichas y en formato PDF.

De las patentes obtenidas tanto en OMPI como en IMPI, se realizó una base de datos propia donde se extrajo la fecha de concesión, el número de solicitud, el solicitante de la patente, el país del solicitante de la patente, el título y resumen de la patente. Al realizar la lectura del resumen se seleccionó si la patente era empleada para el TAR o no.

A partir de las bases de datos se identificaron dos categorías independientes a las proporcionadas por OMPI e IMPI. La primera categoría es el tipo de solicitante, donde se permite analizar quiénes se encargan de realizar las investigaciones e invenciones de las patentes; dicha categoría se obtuvo a partir de la información de la patente en el apartado "solicitante de la patente", se decidió conocer la aportación de cuatro organizaciones o agentes importantes: universidad, centro de investigación, empresa e inventor independiente.

La segunda categoría pertenece al objeto de invención de la patente, la cual determina el alcance de protección de las invenciones, así como ver en qué trabaja la comunidad vinculada al TAR. Se incluyeron cinco modalidades: "equipo", "equipo y procedimiento", "equipo y uso", "procedimiento" y "procedimiento y uso".

Para la selección de estas subcategorías se leyó el resumen de cada patente, en el caso de "equipo", se eligieron los dispositivos o accesorios nuevos o mejorados para el TAR. Para "equipo y procedimiento" 
se seleccionaron las patentes con dispositivos o accesorios nuevos o modificados con una serie de pasos para ejecutarse. En "equipo y uso" se consideraron los dispositivos o accesorios nuevos o mejorados cuya función es utilizarse en TAR, así como en otro proceso. Para la subcategoría de "procedimiento" se seleccionaron aquellas patentes que modificaron una parte de un procedimiento ya conocido. Por último, en "procedimiento y uso" se consideraron las patentes que realizaron una mejora en un proceso que no es para TAR pero se puede utilizar para ello.

\section{RESULTADOS}

\section{Análisis de las patentes para el TAR en OMPI}

Las patentes para TAR tramitadas a través de la OMPI durante el periodo 1996-2013, arrojaron una base de datos con 5041 patentes. Se observa que el flujo de éstas se ha incrementado (Fig. 1a), ya que durante el año de 1996 contaba con 36 invenciones, mientras que para el año 2012 se obtuvieron 634 patentes. Durante 2013, se han registrado 261 patentes, sin embargo, es importante señalar que el número puede ser mayor, ya que existen patentes de invención que se encuentran en estado de solicitud.

El país con mayor número de patentes concedidas, a través de la OMPI, es la República de Corea con 1524 invenciones, lo que representa el $30.23 \%$ de la producción mundial en patentes para TAR, mientras que México cuenta con sólo 34 patentes otorgadas, por lo que su contribución mundial es del $0.67 \%$.

La evolución de las 34 patentes de México en el mismo periodo ha sido aleatoria y además se observa que el patentamiento es nulo en algunos años (Fig. 1b).

De las 34 patentes concedidas a través de OMPI para TAR que se registraron desde México, 20 de éstas pertenecen a titulares extranjeros originarios de Estados Unidos, sólo 7 patentes de las 34 corresponden a titulares mexicanos, por lo que la aportación real de patentes de México es tan sólo de $0.14 \%$.

Durante el periodo 2008-2010, los titulares mexicanos realizaron la mayor aportación de patentes, ya
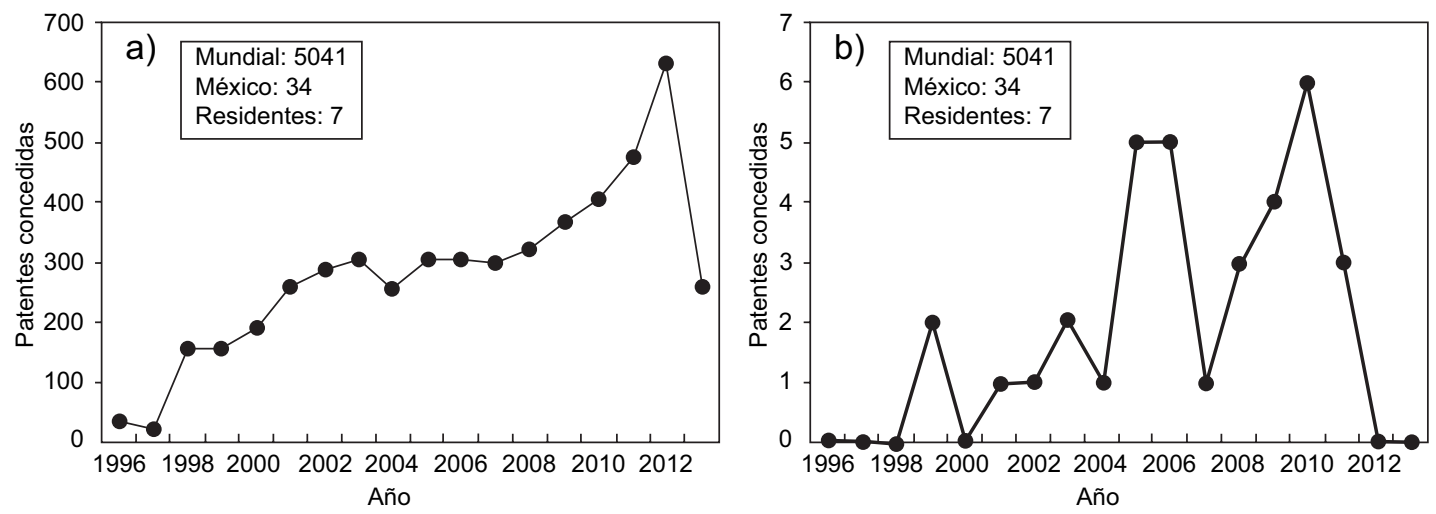

c)

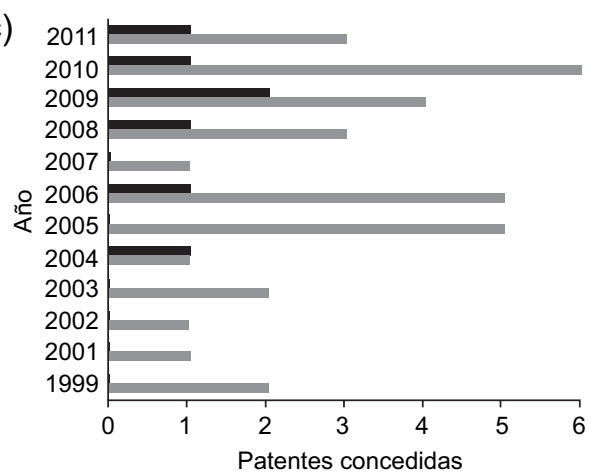

Fig. 1. Evolución dinámica de las patentes tramitadas a través de la Organización Mundial de la Propiedad Intelectual (OMPI) para tratamiento de aguas residuales, 1996-2013: (a) Patentes mundiales, (b) Patentes para México, (c) Patentes concedidas en México por titulares extranjeros comparadas con patentes concedidas en México por titulares mexicanos.

Fuente: Elaboración propia a partir de la base de datos de la OMPI (2014b). 
CUADRO I. PATENTES DE TITULARES MEXICANOS PARA EL TRATAMIENTO DE AGUAS RESIDUALES POR TIPO DE SOLICITANTE Y OBJETO DE PROTECCIÓN

\begin{tabular}{|c|c|c|}
\hline Tipo de solicitante & Objeto de protección & No. patentes \\
\hline $\begin{array}{l}\text { Centro de } \\
\text { Investigación }\end{array}$ & Equipo & 1 \\
\hline Empresa & $\begin{array}{l}\text { Equipo y uso } \\
\text { Procedimiento }\end{array}$ & $\begin{array}{l}1 \\
1\end{array}$ \\
\hline $\begin{array}{l}\text { Inventor } \\
\text { independiente }\end{array}$ & $\begin{array}{l}\text { Equipo } \\
\text { Equipo y procedimiento } \\
\text { Procedimiento y uso }\end{array}$ & $\begin{array}{l}1 \\
1 \\
2\end{array}$ \\
\hline
\end{tabular}

que en esos años se obtuvieron cinco patentes de las siete que tiene registradas México (Fig. 1c).

En la categoría de tipo de solicitante, en el cuadro I, se puede observar que los inventores independientes son los que principalmente realizan dicho trámite, los cuales representan el $57.14 \%$. Al analizar los objetos de protección que los mexicanos trabajan más en cuanto a TAR, se observa que la tendencia es proteger tanto el "procedimiento y uso", así como el desarrollo de nuevos "equipos", ya que cada agrupación tiene dos patentes registradas.

\section{Análisis de las patentes para TAR en IMPI}

En materia de patentes concedidas entre 1996 y 2013, la oficina de patentes de México (IMPI) ha otorgado 137 patentes para TAR. La figura 2a,
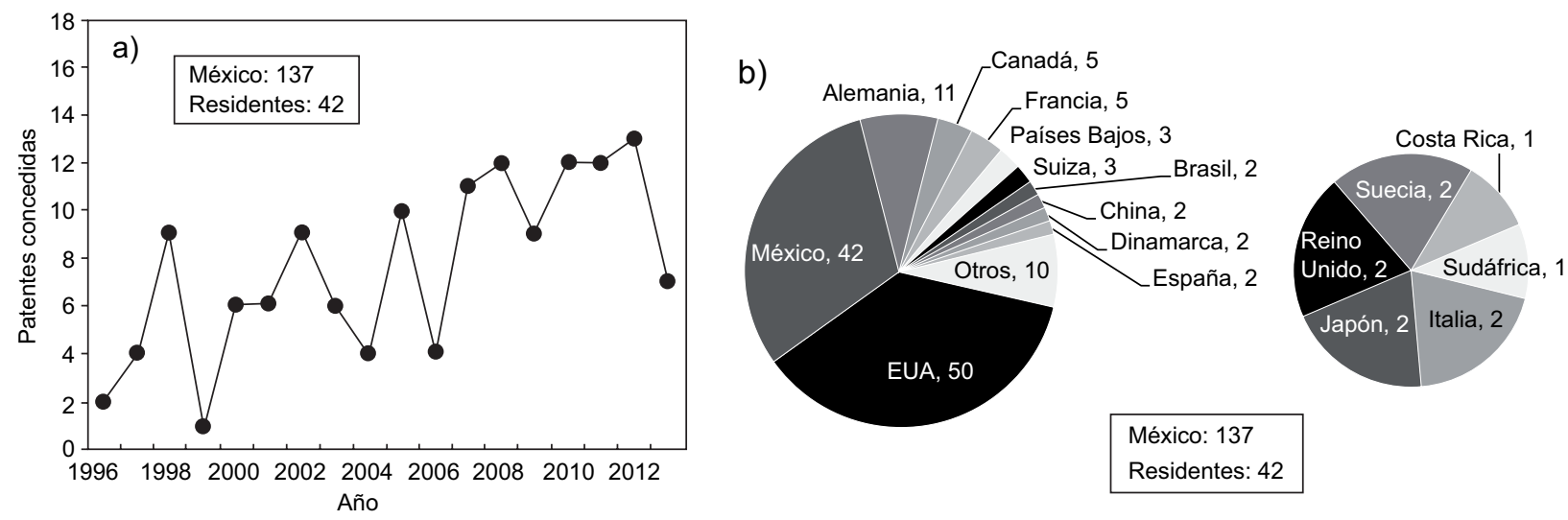

México: 137

Residentes: 42

\section{c)}

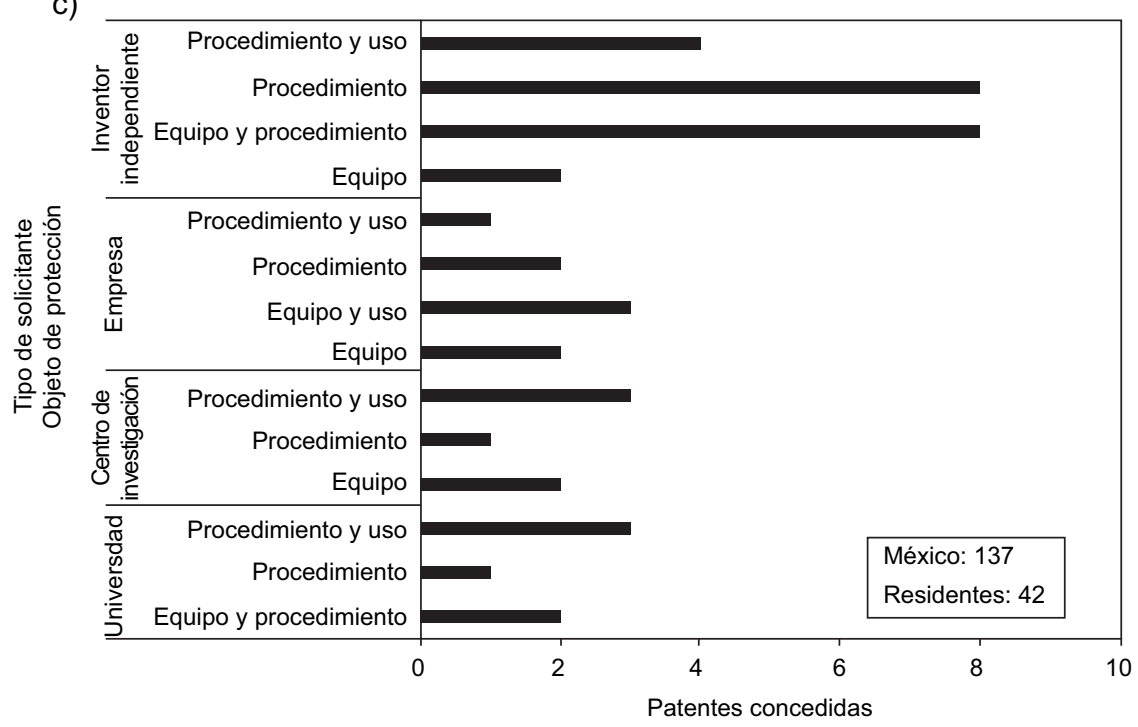

Fig. 2. Evolución dinámica de las patentes concedidas por el Instituto de Mexicano de la Propiedad Intelectual (IMPI) para tratamiento de aguas residuales. 1996-2013: (a) Evolución de patentes por año, (b) Países con patentes concedidas, (c) Patentes concedidas a residentes mexicanos por tipo de solicitante y objeto de protección.

Fuente: Elaboración propia a partir de la base de datos del IMPI (2014a). 
muestra que la evolución de patentamiento en dicho periodo ha sido aleatoria, aunque ha existido un leve incremento en los últimos siete años.

Los resultados de las patentes concedidas (IMPI) muestran que al igual que en la base del OMPI, los extranjeros (no residentes) son quienes cuentan con más patentes con $69.34 \%$ del total, mientras que el $30.66 \%$ restante (42 patentes) pertenece a residentes de nacionalidad mexicana (Fig. 2b).

$\mathrm{El}$ inventor independiente es el principal solicitante de patentes en cuanto a los residentes mexicanos, ya que tiene concedidas 22 patentes de un total de 46. Como se observa en la figura $3 \mathbf{c}$, los principales objetos de protección son el "procedimiento", así como el "equipo y procedimiento". Esta última agrupación es importante para al dueño de la patente ya que le permite tener una mejor transferencia tecnológica.

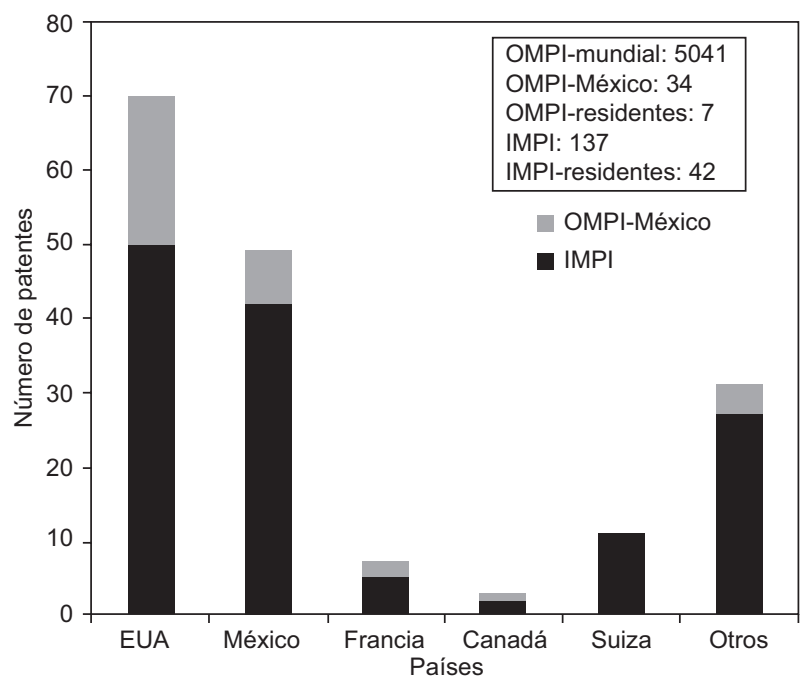

Fig. 3. Comparación de países con patentes tramitadas a través de la Organización Mundial de la Propiedad Intelectual (OMPI) y patentes concedidas por el Instituto Mexicano de la Propiedad Intelectual (IMPI) para México, 19962013.

Fuente: Elaboración propia a partir de las bases de datos del IMPI y de la OMPI (2014a).

\section{Análisis de las patentes para TAR entre OMPI e IMPI}

La OMPI tiene 5041 patentes concedidas durante el periodo analizado, este número comparado con las patentes en el IMPI, que consta de 137, sólo representa $2.72 \%$ de la aportación mundial que investigadores o inventores en México hacen respecto al TAR.

Por otra parte, al separar las patentes que tiene México, según los países del titular tanto la OMPI como el IMPI tienen un papel similar al momento de la concesión de dichas patentes a los titulares extranjeros, como se observa en la figura 3. En ambas bases de datos, se obtuvo que Estados Unidos es el país preponderante, al tener el mayor número de patentes concedidas, mientras que México se encuentra en la segunda posición.

Al analizar las patentes de los titulares mexicanos en las bases de datos de la OMPI como del IMPI, los resultados mostrados en la figura $4 \mathbf{a}$ evidencian que sólo tres patentes están registradas en ambas oficinas, dos de ellas pertenecen a inventores independientes y una a una empresa. El objeto de protección es distinto en las tres, siendo estos: "procedimiento y uso", "equipo y uso" y "equipo" (Fig. 4b).

\section{DISCUSIÓN}

El análisis de este trabajo permite observar que la trayectoria de la tecnología patentada en México para el TAR es escasa en comparación con el volumen y la variedad de las patentes correspondientes a otros países del mundo. De las patentes que México tiene registradas a nivel nacional e internacional, la mayor parte pertenecen a titulares extranjeros, tal como lo muestran los estudios de Aboites y Soria (1999) y Ramírez Guardado et al. (2013). Dichos autores determinaron el perfil tecnológico de patentamiento en el país, así como el otorgamiento de patentes a inventores o empresas extranjeras.

Estados Unidos es el país preponderante al tener el mayor número de patentes solicitadas y concedidas para el TAR en México, ya que a partir del Tratado de Libre Comercio de América del Norte (TLCAN) se volvió la economía con la cual México mantiene más vínculos comerciales, lo que dio lugar a una mayor inversión extranjera directa (Mejía-Reyes et al. 2006). Lo anterior permite llevar a cabo una comercialización importante de tecnología relacionada al TAR.

A pesar de que México cuenta con una instancia para realizar el patentamiento nacional (IMPI), además de encontrarse adscrito al OMPI para el ámbito internacional, se determinó que del componente de tecnología patentada en México sólo 30 \% pertenece al núcleo. Además, son muy pocos los residentes mexicanos que realizan el proceso simultáneo de patentamiento nacional e internacional, esto podría deberse a los costos que tienen las solicitudes de patente, aún cuando en ambos trámites existen descuentos para llevarlos a cabo (IMPI 2014c, IMPI 2014d). 

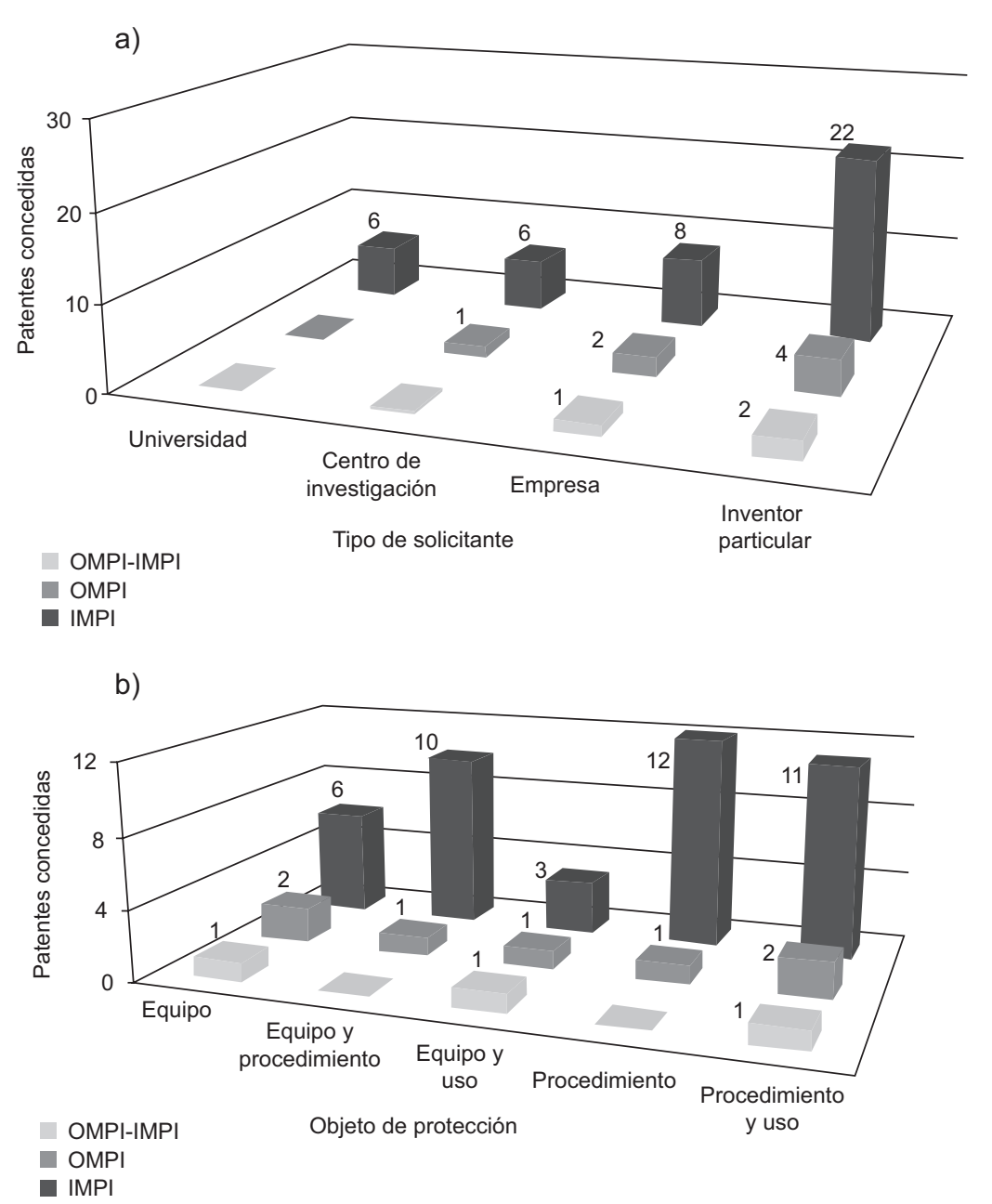

Fig. 4. Patentes concedidas en México por titulares mexicanos a través del Instituto Mexicano de la Propiedad Intelectual (IMPI), la Organización Mundial de la Propiedad Intelectual (OMPI) y el Instituto Mexicano de la Propiedad Intelectual- Organización Mundial de la Propiedad Intelectual (IMPI-OMPI): (a) Tipo de solicitante, (b) Objeto de protección.

Fuente: Elaboración propia a partir de las bases de datos del IMPI (2014a) y de la OMPI (2014b).

Por otra parte, la tecnología patentada de residentes mexicanos desarrollada en centros de investigación y universidades es débil en comparación con los otros solicitantes (empresas e inventores independientes). Esto puede deberse a que el tiempo de concesión de una patente, de acuerdo al IMPI (2014a) es de 4 a 5 años, lo cual es un periodo largo comparado con el tiempo para la publicación de un artículo. Adicionalmente, en el ambiente académico un artículo se considera una mayor aportación a la ciencia y a la tecnología que a una patente, sin tomar en cuenta que el patentamiento es un medio de información para la sociedad sobre los nuevos inventos y los avances tecnológicos que se han realizado hasta hoy en día (Huerta Reyes et al.
2009). Es importante hacer notar que si bien el patentamiento en instituciones de educación superior es poco, el del sector privado también lo es. Se ha documentado que las empresas mexicanas prefieren adquirir tecnología ya probada e importarla. Aunque en el país son las empresas las que más patentan, la mayoría de las ellas son multinacionales que buscan proteger los procesos que las empresas mexicanas reportan (Guadarrama 2011). Además, sólo $5 \%$ del total de las empresas en México realizan investigación y desarrollo (Lino 2013), lo que limita la creación de nuevas patentes. Así que el otro sector que puede realizar dichas actividades de nueva tecnología y patentamiento son las universidades y centros de investigación. Incluso durante el proceso 
de desaparición del modelo nacional de desarrollo de los sexenios 1970-1976 y 1976-1982 existió una política oficial para incentivar la generación de tecnología en instituciones de educación superior y centros de investigación, posiblemente para llenar el vacío de creación de tecnología y patentamiento del sector empresarial.

Los objetos de protección de titulares mexicanos sobre las patentes para el TAR están enfocadas en su mayoría, al desarrollo de "procedimientos" más que al desarrollo de nuevos "equipos", por lo que existe para los investigadores e inventores mexicanos un amplio campo de trabajo en el área de invención para lograr la creación de equipos tecnológicos con mayor eficiencia, menor tamaño y bajo costo. Al igual que en la investigación de Ramos Muñiz et al. (2005) se observa que no existen diferencias significativas en cuanto a la tendencia por objeto de protección. Además, de acuerdo con la evolución de las patentes en el sector del TAR, el núcleo endógeno de tecnología es marginal en México y no hay evidencia de que esté vinculado al flujo global, es decir que se integra escasamente en las redes internacionales de innovación, aunque se requiere de una mayor investigación al respecto (Aboites et al. 2002).

Todo lo anteiror, a su vez, es un fuerte indicador para las autoridades gubernamentales. De hecho, puede ser el motor para implementar programas de estímulos a niveles empresariales y educativos para que apoyen más al TAR con probables resultados prácticos y patentables. Asimismo, sirve para señalar el limitado apoyo que los investigadores han recibido por parte del Gobierno.

\section{CONCLUSIÓN}

La información contenida en los documentos de patentes permite observar cómo ha evolucionado el sector del TAR en el registro de la concesión de patentes en el IMPI y desde México a través del PCT (OMPI), tanto por titulares mexicanos como por titulares extranjeros durante el periodo de 1996-2013.

El volumen de la tecnología patentada en México por titulares extranjeros y mexicanos es escaso en comparación con el volumen de patentes correspondientes a otros países del mundo.

Por ello, el núcleo endógeno de invención de tecnología para el sector del TAR en México es marginal y no hay evidencia de que esté vinculado al flujo global en la materia.

\section{AGRADECIMIENTOS}

Agradecemos al Dr. Rafael Villalobos-Pietrini (Editor), al M. en E. Claudio M. Amescua-García (Editor Ejecutivo), así como a los revisores anónimos de la RICA por su orientación y comentarios que permitieron mejorar significativamente nuestro manuscrito. Uno de los autores (Lilian Edith Domínguez Montero) agradece al Consejo Nacional de la Ciencia y la Tecnología por la beca de posgrado para la realización de esta investigación.

\section{REFERENCIAS}

Aboites J. y Soria M. (1999). Innovación, propiedad intelectual y estrategias tecnológicas: la experiencia de la economía mexicana. Miguel Ángel PorrúaUniversidad Autónoma de México, México D.F., México, 254 pp.

Aboites J. y Soria M. (2008). The production of patented technology by mexican public and private $\mathrm{R}$ and $\mathrm{D}$ Institutions, 1980-2007. Memorias. $3^{\text {a }}$ Conferencia Anual de la Asociación Política Europea de la Propiedad Intelectual. Berna, Suiza. 3 y 4 de octubre, 2008. CD-ROM

Aboites Aguilar J., Soria López M. y Rosado Briseño G. (2002). Trayectorias de patentes y redes de innovación sobre catálisis en México. En: Innovación, confianza y pequeña empresa (M. M. Saleme Aguilar y R. Estrada García, Eds.) Universidad Autónoma Metropolitana, México D.F., México, pp. 23-62.

De la Peña M.E., Ducci J. y Zamora Plascencia V. (2013). Tratamiento de aguas residuales en México. Banco Interamericano de Desarrollo, México D.F., México, $41 \mathrm{pp}$.

Delgado M., Infante M., Abreu Y., García B., Infante O. y Díaz A. (2010). Metodología de vigilancia tecnológica en universidades y centros de investigación. Rev. CENIC Cien. Biol. 41, 1-13.

García B. y Corvo F. (2007). Patent information in concrete corrosion research. Rev. CENIC Cien. Quím. $38,410-414$.

Guadarrama J.J. (2011). Las empresas mexicanas viven una sequía de invenciones [en línea]. http:// www.excelsior.com.mx/2011/08/12/dinero/760414 03/09/2015.

Huerta-Reyes M., Aguilar-Rojas A, Zamilpa A y Tortoriello J. (2009). Conocimientos básicos en propiedad industrial. Una guía para las solicitudes de patente para investigadores mexicanos en el área de estudio de las plantas. Bol. latinoam. Caribe plantas med. aromát. $8,41-51$. 
IMPI (2014a). Base de datos del Instituto Mexicano de la Propiedad Intelectual [en línea]. http://siga.impi.gob. mx/\#busqueda 10/03/2014.

IMPI (2014b). Guía de usuario de patentes y modelos de utilidad [en línea]. http://www.impi.gob.mx/patentes/Paginas/GuiaPatentesModelosUtilidad.aspx $03 / 09 / 2014$.

IMPI (2014c). Guía de usuario: tratado de cooperación en materia de patentes [en línea]. http://www.impi.gob.

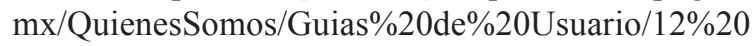
-\%20Tratado $\% 20 \mathrm{de} \% 20$ Cooperaci\%C3\%B3n $\% 20$ en $\% 20$ Materia $\% 20 \mathrm{de} \% 20$ Patentes $\% 20 \mathrm{PCT}$.pdf 03/09/2014.

IMPI (2014d). Tarifas de invenciones, modelos de utilidad, diseños industriales y esquemas de trazado de circuito integrado [en línea]. http://www.impi.gob.mx/patentes/ Paginas/TInvencionesDise \%C3\%B1osCircuitos.aspx 03/09/2014.

Jiménez B. (2006). Irrigation in developing countries using wastewater. Int. Rev. Environ. Strategies. 6, 229-250.

Jiménez B., Durán J. y Méndez J. (2010). Calidad. En: El agua en México: cauces y encauces. (B. Jiménez, M. Torregrosa, L. Aboites, Eds.) Academia Mexicana de Ciencias, México D.F., México, pp. 265-290.

Lin M. (2013). Empresas mexicanas, sólo 5\% hace investigación [en línea]. http://eleconomista.com.mx/ entretenimiento/2013/11/14/empresas-mexicanassolo-5-hace-investigacion-desarrollo 03/09/2015.

Mejía-Reyes P., Gutiérrez-Alva E. E. y Silva C. A. F. (2006). La sincronización de los ciclos económicos de México y Estados Unidos. Investigación Económica. 65, 15-45.

Meyer M., Timm Utecht J. y Goloibeva T. (2003). Free patent information as a resource for policy analysis. World Pat. Inf. 25, 223-231. DOI: 10.1016/S0172-2190(03)00041-3

OMPI (2014a). ¿Qué es una patente? Organización Mundial de la Propiedad Intelectual [en línea]. http:// www.wipo.int/patentscope/es/patents_faq.html\#patent 03/09/2014.
OMPI (2014b). Base de datos de la Organización Mundial de la Propiedad Intelectual [en línea]. http://patentscope.wipo.int/search/en/result.jsf 05/06/2014.

Rendón Cárdenas A.E. (2006). Propiedad intelectual y biotecnología en América Latina: situación, retos y posibilidades. En: Ética e innovación tecnológica. (F. Lolas Stepke, Ed.) Centro Interdisciplinario de Estudios en Bioética, Universidad de Chile, Chile. pp. 123-134.

Ramírez Guardado P., Longar Blanco M., Gómez Víquez H. y Maffini Gomes C. (2013). Política hídrica y cambio tecnológico en tecnologías aplicadas al tratamiento de las aguas residuales. J. Technol. Manage. Innov. 8, 64-75. DOI: $10.4067 / \mathrm{S} 0718-27242013000300035$

Ramos G. y García B. (2005). Tendencias en el desarrollo de nuevas tecnologías para el tratamiento de aguas, residuos y lodos. Rev. CENIC Cien. Biol. 36, 34-46.

Ramos Muñiz G., García Delgado B., Tundidor Camba A. y Bataller M. (2005). Vigilancia tecnológica e información de patentes: Su papel en el enfrentamiento a la sequía. Rev. CENIC Cien. Quím. 36, 52-64.

Romero-Aguilar M., Colín-Cruz A., Sánchez-Salinas E. y Ortiz-Hernández M. L. (2009). Tratamiento de aguas residuales por un sistema piloto de humedales artificiales: evaluación de la remoción de la carga orgánica. Rev. Int. Contam. Ambie. 25, 157-167.

Salgado-Bernal I., Durán-Domínguez C., Cruz-Arias M., Carballo-Valdés M. E. y Martínez-Sardiñas A. (2013). Bacterias rizosféricas con potencialidades fisiológicas para eliminar materia orgánica de aguas residuales. Rev. Int. Contam. Ambie. 28, 17-26.

Vidal R. (2008). Instrumentos de protección intelectual: naturaleza de la patente. Epistemus: Ciencia, Tecnología y Salud 4, 57-65.

Zurita-Martínez F., Castellanos-Hernández O. y Rodríguez-Sahagun A. (2011). El tratamiento de aguas residuales municipales en las comunidades rurales de México. Rev. Mex. Cien. Agríc. 1, 139-150. 